\title{
Gallstone formation associated with weight reduction
}

\author{
JOEL L. DICKERMAN, DO
}

The author presents the case history of an obese patient in whom gallstones developed 5 months after she began a weight-loss program. Conservative management with chenodeoxycholic acid completely resolved cholelithiasis following four months of treatment. Gallstoneformation has traditionally been attributed to supersaturation of bile with cholesterol. However, the author proposes that the formation of gallstones may also be related to a change in gallbladder mechanics stimulated by dieting over an extended period. Recognition of this risk can lead to early diagnosis of gallstones, thus enhancing effective treatment by conservative methods.

Gallstone formation has long been associated with obesity, but exactly how the two are related has been unclear. The following case report describes the development of gallstones in a dieting patient and her subsequent treatment by conservative therapy. The development of gallstones may be related to gallbladder mechanics, making the latter another risk factor for the development of gallstones. Investigation of gallbladder mechanics may shed light on ways to prevent gallstone formation.

\section{Report of case}

A 26-year-old woman.consulted our office regarding desired weight loss. The patient said she had tried without success to diet on her own. When first seen by the author, she weighed $341 \mathrm{lbs}$. Laboratory values revealed a thyroid-stimulating hormone (TSH) level of 9.18 (normal, 0.3 to 5.0) and a serum cholesterol level of $202 \mathrm{mg} / \mathrm{dL}$. Thyroid therapy was started with $0.1 \mathrm{~g}$ of levothyroxine so-

At the time this article was written, Dr Dickerman was in private practice and in emergency medicine at Northwest General Hospital, Milwaukee, Wis.

Reprint requests to Joel L. Dickerman, DO, PO Box 443, Cascade, CO 80809. dium (Synthroid) per day and a 1200 calorie diet was prescribed. The patient was instructed to exe ercise mildly three times a week for 20 minutes.

The patient was followed up weekly. She was very cooperative and consistently lost between 3 and $9 \mathrm{lbs}$ a week. Her thyroid functions were monitored regularly and she was maintained on 0.15 $\mathrm{g}$ of levothyroxine sodium daily.

Approximately 5 months after beginning her weight-loss program, the patient experienced some mild abdominal pain accompanied by diarrhea. She was treated for viral enteritis and instructed to return if symptoms continued. The symptoms disappeared in 2 days.

About 1 month later, the patient again experienced abdominal pain. Physical examination revealed right upper quadrant tenderness, and a detailed patient history revealed increasing fatty food intolerance. Ultrasonography of the gallbladder was performed, but the gallbladder could not be well visualized. Double-dose oral cholecytography revealed a layer of multiple, small cholesterol stones (Fig 1). Because the patient wished to avoid surgery, chenodeoxycholic acid therapy $(1750 \mathrm{mg}$ daily, based on the patient's weight) was instituted. After 4 months of treatment, a second oral cholecystogram revealed that the stones had completely dissolved (Fig 2).

\section{Discussion}

Traditionally, gallstone formation has been attributed to supersaturation of bile with cholesterol. ${ }^{1}$ Normally, there is a balance among bile acids, cholesterol, and lecithin in the gallbladder. With either an increase in cholesterol or a decrease in bile salts, supersaturation of bile can occur. However, Pattinson and Chapman ${ }^{2}$ showed that, even with supersaturation, stone formation may not occur. They found that, in fasting states, when bile salt formation was suppressed, cholesterol remained suspended in a nonmicelle formation. They hypothesized that a cholesterol vesicle might be present which could suspend cholesterol in the presence of low bile salts. This could rule out cho- 


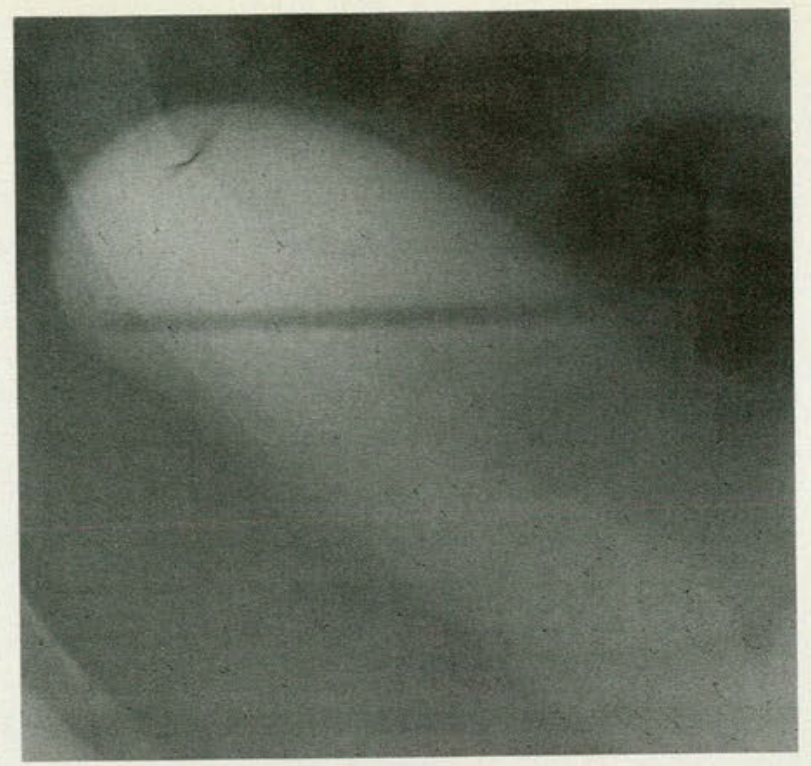

Figure 1. Double-dose oral cholecystogram reveals a fine line of floating cholesterol stones.

lesterol supersaturation as the only factor in gallstone formation.

Perhaps another factor important in gallstone formation is gallbladder kinetics. Long periods of noncontraction of the gallbladder may lead to stone formation. Marzio and associates $^{3}$ studied the kinetics of the gallbladder in both obese and nonobese patients during fasting and nonfasting states. Obese individuals tended to have greater gallbladder volume and fewer gallbladder contractions than nonobese individuals. During fasting, both types of patients showed an increase in resting gallbladder volume. Obese individuals showed this to a greater extent. I believe it is possible that fasting in some obese people leads to fewer gallbladder contractions as well as greater gallbladder volume, thus increasing the risk of gallstone formation.

Gallstone formation has long been associated with pregnancy. Progesterone has an inhibitory effect on smooth muscle contraction and leads to relaxation of the gallbladder. ${ }^{3}$ This fact supports the theory that a decrease in gallbladder contraction may contribute to gallstone formation by a similar mechanism of action. A diminished response to cholecystokinin $(\mathrm{CCK})$ in obese individuals may lead to

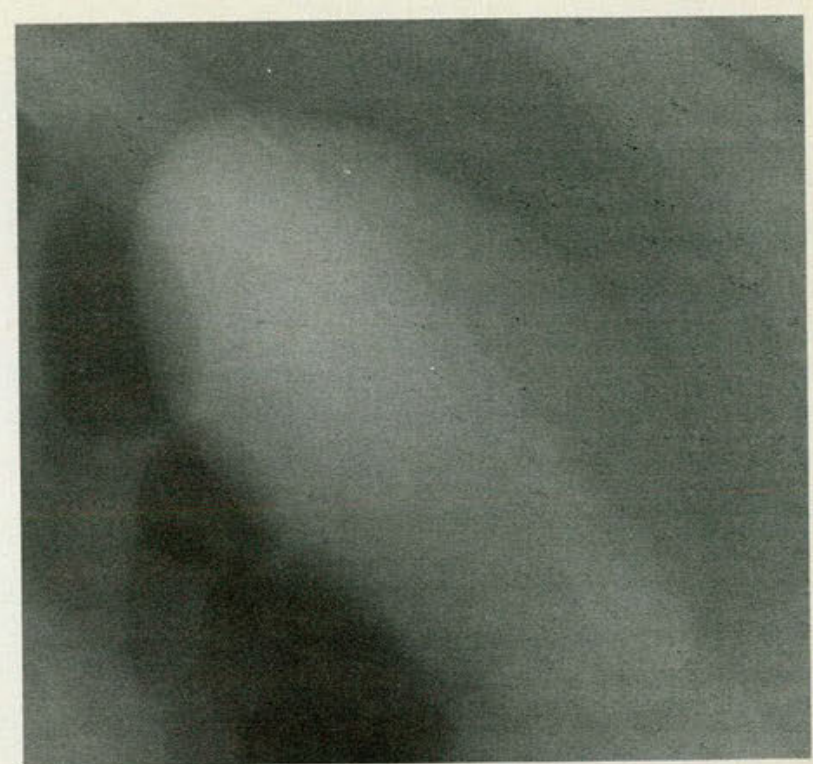

Figure 2. Second oral cholecystogram reveals complete dissolution of stones after 4 months of treatment with chenodeoxycholic acid.

gallbladder stasis. Cholecystokinin is known to suppress feeding because it produces food satiety. Therefore, a diminished output of CCK or a diminished response to CCK may partially account for obesity itself. ${ }^{3}$

The formation of gallstones in the fasting patient may be a valid concern, especially in those individuals at high risk, ie, women, especially those taking oral contraceptives, American Indians, and patients taking clofibrate or gemfibrozil (these lipid-lowering agents increase biliary secretion of cholesterol). ${ }^{3}$ Cholelithiasis should be considered in the differential diagnosis for the fasting patient with nausea, diarrhea, abdominal pain, or food dyscrasias. Since these signs are common in gastroenteritis, food intolerance, and irritable bowel, the possibility of gallbladder disease may be overlooked. Diagnosis of gallstones, if made early, may prevent the complications accompanying acute cholecystitis.

Early detection of gallstones may also permit nonsurgical treatment of the stones. Chenodeoxycholic acid, an orally administered bile salt, is occasionally effective in dissolving cholesterol stones. Small radiolucent stones $(<$ $15 \mathrm{~mm}$ in diameter) and those that float on oral cholecystography have a higher rate of 
dissolution with chenodeoxycholic acid. ${ }^{4}$ Smaller and less numerous stones are also more amenable to ultrasonic dissolution.

\section{Conclusion}

Dieting over an extended period of time may increase the risk of gallstone formation. Fasting leads to decreased bile acid secretion and cholesterol supersaturation of bile, as well as decreased gallbladder contraction and increased biliary stasis. Both of these factors can lead to the development of gallstones, even in the presence of a low-fat, low-cholesterol diet. Recognition of this risk in the dieting individual may lead to early diagnosis of gallstones, precluding the possible complications of acute cholecystitis. Early detection may also permit nonsurgical treatment with stone-dissolving agents or by ultrasonic dissolution.

Further investigation may lead to the formulation of oral bile salt agents that can be added to dietary regimens to inhibit gallstone formation. The addition of small amounts of fat to a low-calorie diet might also prove beneficial in stimulating gallbladder contraction, thereby reducing biliary stasis.

1. Schoenfield J: Gallstones Clinical Symposia, CIBA Geigy Corp, New Jersey, 1988, pp 2-6.

2. Pattinson NR, Chapman BA: Distribution of biliary cholesterol between mixed micelles and nonmicelles in relation to fasting and feeding in humans. Gastroenterology 1986;91:697702.

3. Marzio L, Capone F, Neri M, et al: Gall bladder kinetics of obese patients: Effect of a regular meal and low-calorie meal. Dig Dis Sci 1988;33(1):4-9.

4. Physicians' Desk Reference, Huff B, (ed). Oradell, New Jersey, Medical Economics Co, Inc, 1988, p 1669. 